\title{
EL NUEVO ALUMNO Y EL DESAFÍO DE LA MERITOCRACIA: ANÁLISIS DEL CAMBIO CULTURAL EN LA EDUCACIÓN SUPERIOR CHILENA
}

\author{
Kiyoshi Fukushi ${ }^{1}$
}

\section{RESUMEN}

El siguiente artículo tiene como propósito reflexionar acerca del significativo cambio que ha experimentado el sistema de educación superior chileno en las últimas décadas. Esta reflexión no se enfoca en revisar los cambios en la institucionalidad y sus efectos; sino en relevar al sujeto que ha surgido de dicho proceso. Se trata de un individuo que tiene cabida y sentido en este momento histórico, que asume el desafío que le propone la sociedad; pero al mismo tiempo exige su recompensa: el Chile de las oportunidades no es evidente. Se constata una tensión, por un lado el individuo que acepta la oferta de una formación que le asegure un proyecto de vida, y por otra una elite que no ha "abierto" las puertas para que estos nuevos actores jueguen el rol que esperan. Así, la meritocracia queda en el ámbito de lo pendiente.

Palabras clave: movilidad social, meritocracia, educación superior.

\section{THE NEW STUDENT AND THE MERITOCRACY CHALLENGE: ANALYZING THE CULTURAL CHANGE IN CHILEAN HIGHER EDUCATION}

\begin{abstract}
This article intends to reflect on the significant change experienced by the Chilean higher education system in recent decades. This discussion does not focus on reviewing the institutional changes and their effects but in relieving the subject that has emerged from this process. It is an individual belonging to this historical moment which assumes the challenge that society offers but also demands its reward, since the opportunities in Chile are not evident. It notes a tension - on one hand the individual who accepts the training supply in order to have a life project guaranteed and on the other hand an elite that has not "opened" the doors to these new actors to play the role they expect. Thus, meritocracy still lies as something unresolved.
\end{abstract}

Key words: social mobility, meritocracy, higher education.

1 Vicerrector de Aseguramiento de la Calidad, Universidad San Sebastián, Chile. Contacto: kfukushi@uss.cl 


\section{EL NUEVO ALUMNO Y EL DESAFÍO DE LA MERITOCRACIA: ANÁLISIS DEL CAMBIO CULTURAL EN LA EDUCACIÓN SUPERIOR} CHILENA

¿La nieve que cae

es otra este año?

Bashô

\section{Los efectos del cambio}

El sistema de educación superior en Chile ha vivido profundos cambios a partir de la reforma introducida el año 1981. Dicha reforma permitió la creación de universidades privadas y de instituciones no universitarias orientadas a la formación vocacional, tales como institutos profesionales y centros de formación técnica. Luego de algunos significativos ajustes, los efectos de dicho proceso han sido palpables en el notable incremento de la cobertura que ha experimentado el sistema: en 1957 eran 20.000 los alumnos estudiando en el sistema de educación superior, 55.000 en 1967 , 116.962 en 1980, 249.482 en 1990 hasta llegar a los 848.105 en el año 2010. Sobre la relevancia de este proceso, el Informe que realizó la OCDE el año 2009 sobre la educación superior en Chile, además de elogiar los avances experimentados, señaló:

"[...] Los chilenos valoran la educación; la ven como el camino más importante y seguro hacia una continua prosperidad y hacen grandes esfuerzos, como individuos y como sociedad, para tener acceso a una educación de calidad y aprovechar las ventajas de las oportunidades que ésta brinda". ${ }^{2}$

2 OCDE y Banco Internacional para la Reconstrucción y el Desarrollo / Banco Mundial (2009). 
En la actualidad la "industria" de la educación superior es considerada una de las más competitivas del país. Esto se refleja, por ejemplo, al término de cada año con la extraordinaria publicidad que las instituciones de educación superior realizan con el propósito de cautivar a los miles de estudiantes que rinden anualmente la PSU (Prueba de Selección Universitaria).

El ya mencionado incremento en la cobertura -la que alcanzó el año 2010 a casi un 48\% del grupo etario 18-24- se explica por la ampliación de la oferta de programas y carreras especialmente de las universidad privadas, la mayor ayuda financiera en modalidad de becas y préstamos bancarios en condiciones especiales, ayuda alimenticia, así como también el mayor reconocimiento social que ha tenido la formación vocacional o para el trabajo, que ha validado a los institutos profesionales y centros de formación técnica. También, es necesario incluir como hitos de esta transformación al sistema de aseguramiento de la calidad, evolución en la gestión institucional, esquemas de gobernanza; además de, mecanismos publicitarios y de difusión.

Mucho se ha escrito sobre los efectos y también acerca de la ya señalada institucionalidad que ha dado pie a estos cambios; sin embargo, ¿qué ha pasado con el proyecto de vida de los estudiantes y sus familias?, ¿qué ha pasado con el ciudadano común y corriente?, ¿de qué forma se ha hecho parte de estos cambios? Más allá, de las evidentes transformaciones que ha experimentado el sistema de educación terciario chileno, se debe mirar la realidad cotidiana de aquel individuo que construye su biografía a partir de una oferta que antes estuvo negada para los miembros de su familia: para él ésta es la respuesta que posibilita su movilidad social. Tales son las preguntas que motivan esta reflexión, cuestiones que -en opinión del autor- no han sido abordadas con la misma profusión con que lo han sido las transformaciones estructurales que han favorecido estos avances.

\section{La importancia de la educación superior}

La importancia de la educación superior se constata ante el desafío de la modernidad. Alcanzar el desarrollo y poder competir en un mundo globalizado requiere disponer de un adecuado stock de capital humano 
avanzado. Chile requiere disponer de un número de profesionales y técnicos que den viabilidad a su meta de alcanzar el desarrollo, dotando al país de la competitividad acorde con las exigencias de dicha condición. Así lo afirma Rodríguez-Ponce (2009a):

"En consecuencia, la formación de capital humano
avanzado es un imperativo estratégico que deben
asumir las instituciones universitarias en la sociedad del
conocimiento. Se trata de un requisito fundamental, tanto
si las empresas locales pretenden llegar a otros horizontes
geográficos, como si las empresas multinacionales eligen
disponer sus centros de operación en una economía en
particular. Sin capital humano avanzado, una economía
no puede ser competitiva, ni puede constituirse en un
polo de atracción para la inversión extranjera".

Más aun, de acuerdo con las conclusiones de un estudio realizado por el Instituto Libertad y Desarrollo, ${ }^{3}$ la educación media no protegería a los hogares de volver a caer en la pobreza. La probabilidad de ser pobre incluso con estudios secundarios es casi la misma que la de los jefes de hogares que sólo tenían educación básica. En ese sentido, el culminar la enseñanza básica y secundaria no ayudaría a las personas a incorporarse al mercado laboral. De allí también el imperativo de la formación terciaria.

Es interesante constatar que tales desafíos no son nuevos y que han estado presentes desde los propósitos fundacionales de las primeras instituciones de educación superior del país. Abdón Cifuentes, en la solemne asamblea inaugural de la Pontificia Universidad Católica de Chile allá por 1888, dijo:

"Señores: es preciso fundar en una vasta escala y de una manera científica la enseñanza industrial del pueblo; es preciso abrir nuevos y variados horizontes a sus vocaciones de actividad y de trabajo; es preciso darle una instrucción más aplicable a sus necesidades, es preciso multiplicar los medios de ganar la vida a esos millares de jóvenes...".

3 Ver en Libertad y Desarrollo (2010). 
Incluso antes, en 1836, Andrés Bello se preguntaba:

"¿Qué haremos con tener oradores, jurisconsultos y estadistas, si la masa del pueblo vive sumergida en la noche de la ignorancia, y ni puede cooperar en la parte que le toca en la marcha de los negocios, ni a la riqueza, ni ganar aquel bienestar a que es acreedora la gran mayoría de un estado?".

En el espíritu de los fundadores, tanto de la Universidad de Chile como de la Universidad Católica, reconocidas instituciones de educación superior, estuvo presente el propósito de formar a la gran población del país.

Existe, además, un antecedente cultural que se debe tener muy presente. La educación superior tiene y ha tenido un carácter aspiracional para la familia chilena. Muchos de los padres aspiran que sus hijos lleguen a la educación superior y de ese modo se conviertan en mujeres y hombres que dispongan de las herramientas necesarias que les permita alcanzar una mejor calidad de vida. Así como antes fue aspiracional la enseñanza básica, y luego lo fue la secundaria, hoy lo es la terciaria. Esta aspiración ha sido parte del devenir histórico de la familia chilena más claramente a partir de bien entrada la segunda mitad del siglo XX. Con ese objetivo, madres y padres se esfuerzan día a día por darles la oportunidad a sus hijos para que puedan educarse, de tal forma que la vida les sea distinta a la que les ha tocado vivir a ellos. Según datos de la Comisión Nacional de Acreditación de Chile (CNA-Chile), que ratifican lo afirmado antes por Armanet (2005), en la actualidad siete de cada diez estudiantes que ingresan a la educación superior corresponderían al primer miembro de la familia que lo hace. ${ }^{4}$

Así, las iniciativas que han posibilitado el notable desarrollo de la educación superior, han atendido a inspiraciones que estuvieron latentes desde la fundación de las más antiguas de las instituciones de educación superior del país. También ellas han estado en la íntima aspiración de las familias. Planteado de esa forma, el cambio

4 Ver Rodríguez-Ponce (2009b). 
experimentado en las estructuras y leyes, y como consecuencia en la dinámica actual de la "industria" de la educación superior, ha sido "buscado" (o propuesto) desde antiguo. Entonces lo nuevo se debe reconocer en el profundo e impresionante cambio que han experimentado los individuos, en su reacción frente a estas nuevas oportunidades, posibilidades que en su proyecto de vida hacen una diferencia, que hacen un nuevo comienzo posible.

\section{La subjetividad del "nuevo" alumno}

Ante la transformación que proponía el Estado, por medio de los cambios en la institucionalidad del sistema -primero en los inicios de los 80 y con políticas públicas incorporadas en los 90, que favorecían el acceso masivo a la educación terciaria y luego profundizadas en la primera década del siglo XXI- la sociedad reaccionó en forma positiva. Los alumnos rápidamente aceptaron las nuevas oportunidades que se les ofrecían. Este nuevo alumno, que ve en la educación superior un camino para la movilidad social, comienza, por aquellos años, a ocupar un rol destacado en la definición de la forma en que las instituciones debían interactuar con ellos.

Esta efectiva participación del discente provocó que estas nuevas instituciones privadas se volvieran de interés para el empresariado, el que muy prontamente comenzó a incorporarse en la propiedad y en la gestión de las mismas, trayendo el modelo de administración empresarial al mundo de la educación superior. Los alumnos empezaron a ser considerados como "clientes", en una semántica distinta a la que se había manejado tradicionalmente.

Con todo ello, nuevas vivencias familiares surgieron en la cotidianidad. A modo de ilustración, casos como los de Silvia y Karen comienzan a ser comunes en nuestro país. Los padres de Silvia nunca fueron a la universidad. Su padre trabaja en una fábrica de muebles. Con el esfuerzo de su familia y un par de becas estudiantiles y mucho trabajo duro, Silvia se graduó de profesora hace un par de años. Hoy día trabaja en una escuela rural intentando conectar a sus estudiantes con los recursos que ellos necesitan para ser exitosos: no todos los padres pueden pagar una mensualidad y la sola determinación no 
es suficiente. De esa forma, todos los días Silvia les está dando a sus alumnos la misma oportunidad en educación que ella tuvo.

Karen tiene dos hijos pequeños, vive sola, con su trabajo paga las cuentas. Pero está preocupada por su futuro. Le gustaría estudiar una carrera y construir así un mejor porvenir. La opción para poder estudiar se encuentra a más de 200 kilómetros de distancia de su hogar. Para poder matricularse debería dejar su trabajo, poner a sus hijos en una sala cuna, y en definitiva gastar dinero que no tiene. Karen se entera de un programa que se imparte en formato online. Se matricula. De ese modo, ella continua con su empleo, sabe que pronto tendrá un título... no es fácil pero se está esforzando. Por primera vez en mucho tiempo, Karen puede ver su futuro con optimismo.

Estas biografías nos muestran a un sujeto que tiene sentido sólo en este momento de la historia; es consecuencia del cambio que ha experimentado la educación superior: un sujeto que entiende que su futuro depende de él, que es dueño de su porvenir ${ }^{5}$ y lo asume; un alumno que quiere "el Chile de las oportunidades" que se le ha ofrecido.

Existen al menos dos nuevos tipos genéricos de estudiantes que deben ser considerados para comprender y describir su subjetividad. Estos grupos son los que se han sumado a la elite que tradicionalmente ha accedido al sistema.

El primero de ellos se relaciona con una clase media emergente que hoy día tiene una amplia posibilidad de acceso a la educación superior. Estos alumnos representan a familias que por primera vez uno de sus miembros le tuerce la mano a su historia familiar y puede, por sus méritos, aprovechar una oportunidad que le "promete" un cambio sustantivo en su vida.

Además, existe otro creciente grupo de estudiantes a tener en cuenta. Cada vez son más los trabajadores que buscan y encuentran en las instituciones de educación superior una posibilidad de mejorar sus condiciones laborales y se inscriben en ellas buscando la obtención

5 Ver Beck (2001) y Beck y Gersheim-Beck (2003). 
de un título profesional. Este grupo será cada vez más importante para las instituciones de educación superior. ${ }^{6}$

Entre las características más relevantes en la subjetividad del estudiante que surgen de este cambio se destacan las seis siguientes:

(i) Su proyecto de vida incluye la formación terciaria. Se está transitando desde una educación de elite a una masiva. El notable crecimiento de la cobertura en la clase media demuestra que el impedimento para acceder al sistema de educación superior está reducido al interés personal y a la capacidad de enfrentar los estudios;

(ii) Es exigente y reclama métodos pedagógicos que reconozcan su condición sociocultural. La convicción de que "estoy pagando por un servicio" los fortalece en sus exigencias. Ello obliga a pensar sobre el tipo de sistemas educativos requeridos en este nuevo contexto. Cuando se considera a aquellos trabajadores que ven la alternativa de mejorar su condición laboral accediendo al sistema de educación superior, se debe trabajar en los métodos pedagógicos más apropiados. Se trata de responder a preguntas tales como ¿qué experiencias educativas puede formar la vida de una persona que ya tiene una biografía personal avanzada?, ¿cómo enseñar a personas con su propio contexto de vida y cultura?, ¿son adecuadas las metodologías que usamos para enseñarles?, ¿son los formatos adecuados?, ¿su velocidad de aprendizaje y su contexto son reconocidos por los actuales planes de estudio?;

(iii) Preocupación por su comunidad. Les interesan las actividades de voluntariado, de ayuda social. El año 2009 la Escuela de Periodismo de la Universidad Diego Portales (Chile) y la empresa Feedback dieron a conocer los resultados de un estudio sobre la participación social de jóvenes ${ }^{7}$, aplicada a hombres y mujeres

6 Típicamente el público objetivo para éstas se sitúa en el grupo de jóvenes entre 18 y 24 años. De acuerdo con el INE (Instituto Nacional de Estadísticas), este grupo crecerá hasta el año 2015, llegando a alrededor de 1,5 millones de personas; a partir de ese año el grupo comenzará a disminuir, y "a mediano y largo plazo la población que demandará educación superior provendrá crecientemente de los grupos de edad mayores a los 25 años", tal como concluye Brunner (2009). Se estima que un joven habrá tenido en promedio 14 empleos antes de cumplir los 38 años, y en consecuencia requerirá de una educación continua.

7 Ver encuesta UDP-Feedback. 
entre 18 y 29 años. Entre los resultados, se destaca el interés manifestado por participar en actividades de voluntariado social y de protección al medioambiente. Si bien es cierto que un 18\% y un $19 \%$ respectivamente reconocían estar participando al momento de responder, un 54\% y un 55\% afirmaron estar dispuestos a hacerlo en el futuro;

(iv) No es un receptor pasivo. Por el contrario es uno informado que cuenta con una enorme cantidad de fuentes de información, que corrobora y aclara sus dudas en tiempo real a través de Google u otras herramientas. Las vías de comunicación que estos estudiantes utilizan son también muy relevantes, y lo son en particular porque su uso determina el grado de conexión que estos individuos tienen con sus pares, y con ello obtienen un "respaldo" o "apoyo" para su actuar; así estos estudiantes son fuertes consumidores de lo que se conoce como "redes sociales": se trata en definitiva de a quiénes conoces, qué puedes hacer con ellos, el conocimiento contingente que adquieres vía esta conexión. Tanto Facebook como Twitter constituyen dos medios fuertemente utilizados;

(v) Buscan una imagen propia, que los distinga. Les resulta de la mayor importancia crear una identidad personal e imagen propia; quieren un sello que los distinga como sujetos. Existe una fuerte consideración de valor asociada a cómo son percibidos por el otro, ${ }^{9}$ de allí que el uso de tatuajes, aros, pulseras, peinados responde a definir una individualidad distintiva;

(vi) Creen y buscan las oportunidades. Para ellos las "oportunidades" están ligadas a abrir nuevos mundos, explorar, desafiar, descubrir y crecer; pero también a ayudar a otros a crecer. ${ }^{10}$ Quieren que las oportunidades se traduzcan en la factibilidad cierta de contratación al egreso; sueldos equivalentes al de los pares para funciones similares; una "contienda justa", en el sentido de que se compita de igual a igual, que se evalúen las competencias de cada uno y no factores extras; también estabilidad laboral, condiciones

8 En la misma encuesta UDP-Feedback los jóvenes señalan que un 71,3\% de ellos está registrado en Facebook y un 47,6\% lo visita todos los días.

9 Ver Asokan, Ashwini: "And they told me i needed more math". En Sungard Summit Connect 2009. Philadelphia, USA. http://www.sungarhe.com

10 Ibídem. 
contractuales en igualdad de condiciones en leyes sociales, salud, entre otros.

En definitiva, se está en presencia de un sujeto que quiere ser parte del desarrollo económico que ha experimentado Chile, recibir, también, los beneficios que ello supone. Un sujeto que se hace cargo de su futuro, que no se rige por ideologías, sino que está dispuesto a protestar cuando percibe que el camino a su meta se ve amenazado. ${ }^{11}$ Este nuevo sujeto quiere su espacio; sólo espera que su esfuerzo se vea recompensado en su mérito.

\section{El premio al mérito: camino de movilidad social}

Cuáles son las preocupaciones del estudiante en este nuevo escenario, en el que se le ha afirmado que el futuro sólo depende de él.

Un aspecto de la mayor preocupación para los alumnos es tener la seguridad de que lo que están estudiando les va a garantizar encontrar un trabajo luego de finalizar su formación. Han existido lamentables experiencias en que algunas instituciones han ofrecido programas que no cuentan con un campo laboral verdadero. Por ello, les interesa certificar la calidad de la institución en la que se están matriculando; en otras palabras, que ésta demuestre un reconocimiento social que garantice iguales o mejores condiciones que otras instituciones. Por ello cuando se les pregunta respecto de las razones que los motivaron a matricularse, aparece de interés el

11 Las mayores protestas y movilizaciones estudiantiles registradas han estado motivadas por exigencias hacia la calidad de la educación. Ése fue el objetivo de la protesta realizada en el año 2006, conocida como "protesta de los pingüinos", cuyos puntos máximos se produjeron (el 30 de mayo y el 5 de junio), cuando marcharon 790.000 estudiantes y cuando una huelga nacional convocada por la Asamblea Coordinadora de Estudiantes paralizó el sistema de educación en el país. La mayoría de las universidades cerró y el Colegio de Profesores se declaró también en huelga. Las razones de los estudiantes fueron ampliamente respaldadas por la comunidad y reconocidas como justas: alrededor del $87 \%$ de los chilenos encuestados dijo que apoyaba el movimiento, y los alumnos de colegios privados le dieron su apoyo activo. Aunque los orígenes están claramente en la educación básica y secundaria, las repercusiones traspasaron a todos los niveles, alcanzando claramente a la educación superior. El 13 de noviembre del 2007 se firmó el "Acuerdo para la Calidad de la Educación", que fijó como principios prioritarios del sistema educacional chileno la calidad, igualdad, rendición de cuentas y transparencia. 
constatar que la institución se encuentre acreditada ${ }^{12}$, y no habría razón para no pensar que tal condición se ampliará al programa mismo. Además, les importa saber si el plan de estudios que se está cursando está actualizado respecto de los avances experimentados; si los académicos son idóneos para impartir las asignaturas; y si la carrera está preocupada por lo que va a ocurrir con ella en cinco e incluso diez años más.

Pero una cuestión central en esta transición es que la elite tradicional del país abra la puerta para aceptar a estos nuevos profesionales y técnicos. Aquel alumno que se esforzó con el apoyo de su familia, una vez egresado -en muchos casos con una cuantiosa deuda a cuestas-, quiere su recompensa: quiere ese futuro prometido, feliz y realizador que se le ofreció. Sin embargo, la meritocracia y la movilidad social en Chile siguen siendo un tema pendiente ${ }^{13}$. La elite tradicional ha operado fundamentalmente como una cerrada red de interrelaciones ${ }^{14} \mathrm{y}$, aunque algunos avances ha habido, esa modalidad sigue vigente hasta nuestros días. Querer ser "admitido" en ese círculo en base a los méritos propios tiene la contracara de la frustración y la impotencia social. Los nuevos profesionales se ven enfrentados a un verdadero "cuello de botella" social. El riesgo de la pregunta ¿qué se gana con esforzarse si la recompensa era una promesa incumplida? está latente cuando "el Chile de las oportunidades" no es más que un relato sin sustento. Ése es el gran desafío de la sociedad chilena: abrir las puertas para que ocupen su lugar aquellas personas capaces, esos nuevos sujetos de la educación superior que tienen los méritos

12 La Universidad San Sebastián, durante el año 2008, encargó a la consultora de marketing O’Bois (http://www.obois.cl) la realización de un estudio que ofreciera un panorama respecto tanto de quienes ya estudiaban en la universidad, como también de los potenciales nuevos alumnos. El estudio abarcó las regiones Metropolitana, Bíobío, de Los Ríos y de Los Lagos. Interesaba conocer, entre una gran cantidad de temas, cuáles eran las características de la institución en las que se fijaban para tomar la decisión de matrícula. En general, las respuestas obtenidas incluyeron, entre las razones más señaladas, la condición de ser una institución acreditada. En el caso de aquellos estudiantes provenientes de los establecimientos municipalizados de la Región del Bíobío, dicha cualidad fue la más indicada.

13 Ver Núñez y Miranda (2010).

En Núñez y Risco (2004) se concluye: "Así, este hallazgo indica que Chile no sólo posee una desigual distribución de sus ingresos, hecho ya bien documentado, sino también elevados índices de persistencia en la condición socioeconómica de padres e hijos, lo que puede interpretarse como una desigual distribución de las oportunidades".

14 Ver Jocelyn-Holt (1997). 
pero no las redes. Este "nuevo" chileno quiere ser reconocido por su capacidad y con ello obtener el estatus que le corresponde.

Un estudio realizado por Subjetiva para la Corporación Giro País ${ }^{15}$ concluyó que el 92,3\% de los consultados está de acuerdo con el hecho de que tener familiares o amigos influyentes sigue siendo muy importante para lograr el éxito laboral; igualmente, el 68,6\% señaló su acuerdo con la premisa "Si dos profesionales ingresaran a la misma empresa, en cargos idénticos, siempre llegará más lejos el que proviene de una familia más rica". No obstante, el 83,5\% está de acuerdo con que, actualmente, si una persona se esfuerza y trabaja duro puede mejorar significativamente su situación económica. Hoy más que ayer está latente el deseo de cambiar el status quo por medio de la meritocracia. El desafío es que se abran definitivamente las puertas y se den los espacios para que las oportunidades sean verdaderas.

\section{Conclusión}

En la actualidad, los grandes temas que preocupan a la educación superior en Chile están relacionados con mejorar la institucionalidad en aspectos tales como el financiamiento de las universidades estatales, en lo que se ha nominado "el nuevo trato". Otros desafíos son reformas a las ayudas financieras para alcanzar un sistema único de créditos y becas, ampliación del programa de Becas Chile para que se incluyan en los programas de posgrado chilenos, y una mayor transparencia respecto de la información sobre la acreditación de las instituciones. Sin duda, todos estos temas son muy relevantes; sin embargo, se reconoce un cambio real, pero no total. Se echan de menos acciones que se enfoquen en responder a las particularidades del nuevo estudiante: apoyos para una mejor orientación vocacional; ayudas ya no sólo para el ingreso, sino también para permanecer y lograr la titulación (para ello es imperativo que las instituciones se hagan cargo de las carencias formativas que traen algunos de los estudiantes al ingresar); creación de formatos que permitan combinar el estudio y el trabajo, etc.

15 Estudio publicado en marzo de 2008, disponible en: http://bligoo.com/media/users/1/94426/ images/uploads/estudio-giro-pais-conferencia-prensa-editada-12-marzo-2008-definitiva. pdf 
En especial, faltan iniciativas que tengan como meta favorecer la inserción laboral a las nuevas generaciones, catalizando decididamente la movilidad social. Darles espacios a las nuevas generaciones de profesionales constituye un reto ineludible. Para que ello sea posible, es necesario enfocar los criterios de selección laboral en las cualidades objetivas de los postulantes, no privilegiando la lógica de las redes de influencia. Dichas categorías ya no hacen justicia a la realidad de este tiempo y sólo provocan que persista en la sociedad chilena el imperio de la elite en desmedro del mérito.

El gran aumento en la cobertura del sistema de educación superior se explica especialmente por las universidades privadas y las instituciones vocacionales. Alumnos capaces, profesionales talentosos y meritorios egresan de sus aulas. Por ello es un contrasentido no aprovechar esas cualidades. Aún queda camino por andar para generar la contracara del cambio cultural que ha experimentado el estudiante de nuestro tiempo, éste es, que los espacios laborales valoren las cualidades y el mérito del sujeto al momento de la selección laboral sin discriminar positivamente en torno a factores de pertenencia a una cierta clase social o grupo de influencia; y con mayor razón a la hora de decidir un ascenso. Para que tenga sentido el esfuerzo que ha implicado avanzar en el acceso equitativo a la educación superior, el cambio debe incluir la respuesta al anhelo ofrecido y luego perseguido por esas familias: la movilidad social y una meritocracia real.

\section{Referencias Bibliográficas}

Armanet, P. (2005) Formación universitaria para el siglo XXI. En Foco, n. ${ }^{\circ}$ 44, Expansiva.

Beck, U. (2001) "Vivir nuestra propia vida en un mundo desbocado: individuación, globalización y política". En Giddens, A. y Hutton, W. (eds.). En el límite. La vida en el capitalismo global. Barcelona: Tusquets Editores.

Beck, U. y Gersheim-Beck, E. (2003) "La individualización. El individualismo institucionalizado y sus consecuencias sociales y políticas"; Paidós, Buenos Aires.

Brunner, J. J. (2009) "Proyecciones demográficas: Impacto sobre la educación superior" en: http://mt.educarchile.cl/MT/jjbrunner/archives/2009/03/ proyecciones_de.html 
Encuesta UDP-Feedback http://feedback.cl/publicaciones-feedback/99encuestajovenesfeedbackudp.html?652759ed73bdc089a63ec8ad57 ae8351=3c867181f0827550827cbc3316flfdc5

Jocelyn-Holt Letelier, Alfredo (1997) El peso de la noche: Nuestra frágil fortaleza histórica; Buenos Aires: Ariel.

Libertad y Desarrollo (2010) http://www.lyd.com/lyd/centro_doc/documents/ tp-971-vulnerabilidad\%20social\%20mas\%20alla\%20de\%20la\%20 pobreza-25-06-2010.pdf

Núñez, J. y Risco, C. (2004) Movilidad intergeneracional del ingreso en Chile. En Foco, Expansiva.

Núñez, J. y Miranda L. (2010) Movilidad Intergeneracional del Ingreso y la Educación en Chile. En Coloquio CPCE, Facultad de Educación UDP, Mayo 2010.

OCDE y Banco Internacional para la Reconstrucción y el Desarrollo / Banco Mundial (2009) La educación superior en Chile.

Rodríguez-Ponce, E. (2009a) Las universidades en la sociedad del conocimiento. En Desafíos y perspectivas de la dirección estratégica de las instituciones universitarias. Ediciones CNA-Chile. Santiago, Chile.

Rodríguez-Ponce, E. (2009b) Rendición de cuenta pública de la Comisión Nacional de Acreditación. Ediciones CNA-Chile.

Recibido: 03/09/2010

Aceptado: 08/11/2010 